\title{
Experimental demonstration of the topological surface states protected by the time-reversal symmetry
}

 \\ Wang, ${ }^{2}$ Haijun Zhang, ${ }^{2}$ Xi Dai, ${ }^{2}$ Zhong Fang, ${ }^{2}$ Xincheng Xie, ${ }^{2}$ and Qi-Kun Xue ${ }^{1,2, \text {, }}$ \\ ${ }^{1}$ Department of Physics, Tsinghua University, Beijing 100084, China \\ ${ }^{2}$ Institute of Physics, Chinese Academy of Sciences, Beijing 100080, China
}

(Dated: October 29, 2018)

\begin{abstract}
We report direct imaging of standing waves of the nontrivial surface states of topological insulator $\mathrm{Bi}_{2} \mathrm{Te}_{3}$ by using a low temperature scanning tunneling microscope. The interference fringes are caused by the scattering of the topological states off $\mathrm{Ag}$ impurities and step edges on the $\mathrm{Bi}_{2} \mathrm{Te}_{3}(111)$ surface. By studying the voltage-dependent standing wave patterns, we determine the energy dispersion $E(k)$, which confirms the Dirac cone structure of the topological states. We further show that, very different from the conventional surface states, the backscattering of the topological states by nonmagnetic impurities is completely suppressed. The absence of backscattering is a spectacular manifestation of the time-reversal symmetry, which offers a direct proof of the topological nature of the surface states.

PACS numbers: 68.37.Ef, 73.20.-r, 72.10.Fk, 72.25.Dc
\end{abstract}

The strong spin-orbital coupling in a certain class of materials gives rise to the novel topological insulators in two [1, 2] and three dimensions [3, 4, 5, 6, 7] in the absence of an external magnetic field. The topological states on the surfaces of three dimensional (3D) materials have been studied recently in $\mathrm{Bi}_{1-x} \mathrm{Sb}_{x}$ 6, 8, [9], $\mathrm{Bi}_{2} \mathrm{Te}_{3}$ and $\mathrm{Bi}_{2} \mathrm{Se}_{3}$ 17, 10, 11, 12, 13], which possess insulating gaps in the bulk and gapless states on surfaces. The surface states of a $3 \mathrm{D}$ topological insulator comprise of an odd number of massless Dirac cones and the crossing of two dispersion branches with opposite spins is fully protected by the time-reversal-symmetry at the Dirac points. Such spin-helical states are expected to bring forward exotic physics, such as magnetic monopole [14] and Majorana fermions 15, 16]. To date, the experimental study of topological insulators is predominantly limited to the determination of their band structure by angle-resolve photoemission spectroscopy (ARPES) 8, 9, 10, 11, 12, 13. . Distinct quantum phenomena associated with the nontrivial topological electronic states still remain unexplored. Particularly, there is no direct experimental evidence for the time reversal symmetry that protects the topological property. Here, using the low temperature scanning tunneling microscopy (STM) and spectroscopy (STS), we report the direct observation of quantum interference caused by scattering of the 2D topologically nontrivial surface states off impurities and surface steps. Our work strongly supports the surface nature of the topological states, and provides a way to study the spinor wave function of the topological state. More significantly, we find that the backscattering of topological states by a nonmagnetic impurity is forbidden. This result directly demonstrates that the surface states are indeed quantum-mechanically protected by the time reversal symmetry.

The interference patterns in STM experiments 17, 18,
19, 20] result from the 2D surface states perturbed by surface defects. A surface state is uniquely characterized by a $2 \mathrm{D}$ Bloch wave vector $\vec{k}$ within the surface Brillouin zone (SBZ). During elastic scattering, a defect scatters the incident wave with a wave vector $\vec{k}_{i}$ into $\vec{k}_{f}=\vec{k}_{i}+\vec{q}$, with $\vec{k}_{i}$ and $\vec{k}_{f}$ being on the same constant-energy contour (CEC). The quantum interference between the initial and final states results in a standing wave pattern whose spatial period is given by $2 \pi / q$. When the STM images of a standing wave are Fourier transformed [21], the scattering wave vector $\vec{q}(\hbar \vec{q}$ is the momentum transfer) becomes directly visible in the reciprocal space. In contrast, for bulk states, there will be continuous ranges of wave vectors on the projected SBZ for a given energy. Usually, no distinct interference fringe can be produced by bulk states and visualized by STM. In this sense, the standing wave is surface-states-sensitive and particularly suitable for studying topological insulators.

Our experiments were conducted in an ultra-high vacuum low temperature (down to $0.4 \mathrm{~K}$ ) STM system equipped with molecular beam epitaxy (MBE) for film growth (Unisoku). The stoichiometric $\mathrm{Bi}_{2} \mathrm{Te}_{3}$ film, a robust topological insulator, was prepared on single crystal substrate $\mathrm{Si}(111)$ by MBE. Details of sample preparation are described elsewhere [22]. Shown in Fig. 1(a) is a typical STM image of the $\mathrm{Bi}_{2} \mathrm{Te}_{3}$ film with a thickness of $\sim 100 \mathrm{~nm}$. The atomically flat morphology of the film is clearly observed. The three steps seen in Fig. 1(a) all have the height $(0.94 \mathrm{~nm})$ of a quintuple layer. The steps are preferentially oriented along the three close-packing ([100], [110] and [010]) directions. The image with atomic resolution [Fig. 1(b)] exhibits the two-dimensional hexagonal lattice structure of the Te-terminated (111) surface of $\mathrm{Bi}_{2} \mathrm{Te}_{3}$. Our STM observation further reveals a small density of clovershaped defects on the surface (see supporting material 23]). Simi- 
a

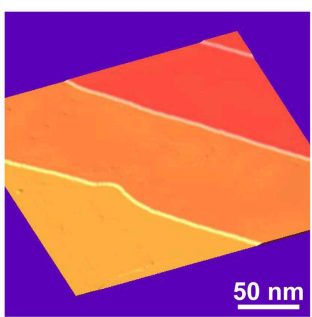

c

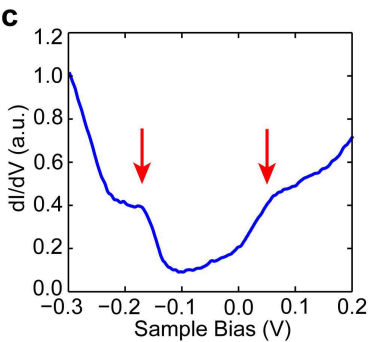

b

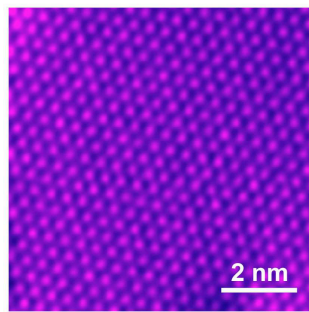

d

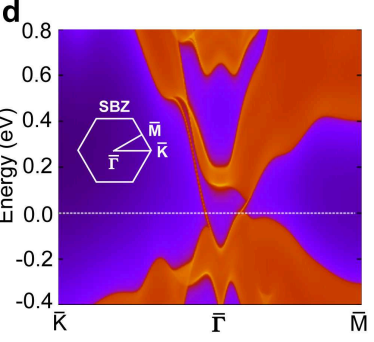

FIG. 1: (a) The STM topograph of the $\mathrm{Bi}_{2} \mathrm{Te}_{3}$ (111) film. The imaged area, $250 \mathrm{~nm}$ by $250 \mathrm{~nm}$, was scanned at a sample bias of $3 \mathrm{~V}$ and tunneling current of $50 \mathrm{pA}$. (b) The atomicresolution image. Tellurium atom (pink colored) spacing is about $4.3 \AA$. The image was scanned at a sample bias of $40 \mathrm{mV}$ and tunneling current of $0.1 \mathrm{nA}$. (c) $d I / d V$ spectrum taken on bare $\mathrm{Bi}_{2} \mathrm{Te}_{3}(111)$ surface. The spectrum was measured with setpoint $\mathrm{V}=0.3 \mathrm{~V}, \mathrm{I}=0.1 \mathrm{nA}$. The arrows indicate the bottom of conduction band and the top of valence band, respectively. (d) Calculated band structure of $\mathrm{Bi}_{2} \mathrm{Te}_{3}(111)$ along high-symmetry directions of SBZ (see the insert). The red regions indicate bulk energy bands and the purple regions indicate bulk energy gaps. The surface states are red lines around the $\bar{\Gamma}$ point.

lar to $\mathrm{Bi}_{2} \mathrm{Se}_{3}[13,24,25]$, these structures can be assigned to the substitutional Bi defects at the Te sites by examining their registration with respect to the $1 \times 1$-Te lattice in the topmost layer.

The surface states of $\mathrm{Bi}_{2} \mathrm{Te}_{3}$ were investigated by STS and the first-principles calculations [7]. The STS detects the differential tunneling conductance $d I / d V$ [Fig. 1(c)], which gives a measure of the local density of states (LDOS) of electrons at energy eV. The two shoulders indicated by arrows in Fig. 1(c) are the bottom of the bulk conduction band and the top of the bulk valence band, respectively. The Fermi level (zero bias) is within the energy gap, indicating that the film is an intrinsic bulk insulator 222]. The differential conductance in the bulk insulating gap linearly depends on the bias and is attributed to the gapless surface states. These features in STS are in good agreement with those obtained by the first-principles calculations (see supporting material [23]). According to the calculations [Fig. 1(d)], the topological states of $\mathrm{Bi}_{2} \mathrm{Te}_{3}$ form a single Dirac cone at the center ( $\bar{\Gamma}$ point) of the SBZ $[10,11]$, giving rise to a vanishing DOS in the vicinity of $k=0$. However, the surface states around $\bar{\Gamma}$ point overlap in energy with the bulk valence band. For this reason, the Dirac point is

invisible in STS.

On the aforementioned surface, we deposited a small amount (0.01 ML) of Ag atoms, which form trimmers on the surface, as shown in Fig. 2(a) and more clearly in supporting material 23]. The atomically resolved STM image (see supporting material [23]) reveals that the $\mathrm{Ag}$ atom in a trimmer adsorbs on the top site of a surface Te atom [26]. This situation is schematically shown in Fig. 2(b). In addition, the Fermi level shifts upwards in energy by $20 \sim 30 \mathrm{meV}$ after $\mathrm{Ag}$ deposition, suggesting electron transfer from the $\mathrm{Ag}$ atoms to the substrate (see supporting material [23]). The $d I / d V$ mapping was then carried out in a region containing $\mathrm{Ag}$ trimmers. At each data point, the feedback was turned off and the bias modulation was turned on to record $d I / d V$. This procedure resulted in a series of spatial mapping of LDOS at various bias voltages.

Figures 2(c) to $2(\mathrm{~g})$ summarize the $d I / d V$ maps for bias voltages ranging from $50 \mathrm{mV}$ to $400 \mathrm{mV}$ from the area shown in Fig. 2(a). The first striking aspect of these images is the existence of standing wave [17, 18, 19] in the vicinity of the $\mathrm{Ag}$ trimmers. The spatial modulation of LDOS by an Ag trimmer forms a hexagonal pattern, whose edges are perpendicular to the $\bar{\Gamma}-\bar{M}$ directions in SBZ. This situation is more clearly resolved at large bias voltages [Figs. 2(f) and 2(g)]. As expected, the interference pattern is anisotropic as a result of the hexagram CEC [10]. The spatial period of the standing wave scales inversely with the bias voltage and is determined by the momentum transfer during scattering at a given energy. Below $50 \mathrm{meV}$, the fringes become obscured. It results from a combination of two effects: (i) The wavelength increases rapidly as the bias voltage approaches the Dirac point, where $k=0$. (ii) At low energy, more topological surface states with different wavelengths are involved in the formation of standing wave as indicated by the firstprinciples calculations [Fig. 1(d)]. The superposition of waves with various wavelengths smears out the interference fringes. With increasing bias, especially after the surface states in the $\bar{\Gamma}-\bar{M}$ direction merge into the bulk conduction band at $\sim 0.2 \mathrm{eV}$ above the Dirac point according to calculation [Fig. 1(d)], the contribution of states in the $\bar{\Gamma}-\bar{M}$ direction vanishes and the states in the vicinity of $\bar{\Gamma}-\overline{\mathrm{K}}$ direction gradually gain more weight, leading to more distinct interference patterns. After the surface states in the $\bar{\Gamma}-\overline{\mathrm{K}}$ direction merge into the bulk conduction band at $\sim 0.6 \mathrm{eV}$ above the Dirac point [Fig. $1(d)]$, the standing waves fade out again.

To quantify the standing waves and obtain the scattering wave vectors, we performed Fourier transformation of the $d I / d V$ maps into the $\vec{q}$-space [Figs. 2(h) to 2(l)]. One important feature in the power spectra can be immediately discerned by comparing the six-fold symmetric pattern in the $\vec{q}$-space with SBZ (the red hexagon in Fig. 2(h)): the regions with high intensity are always oriented toward the $\bar{\Gamma}-\bar{M}$ directions, while the 

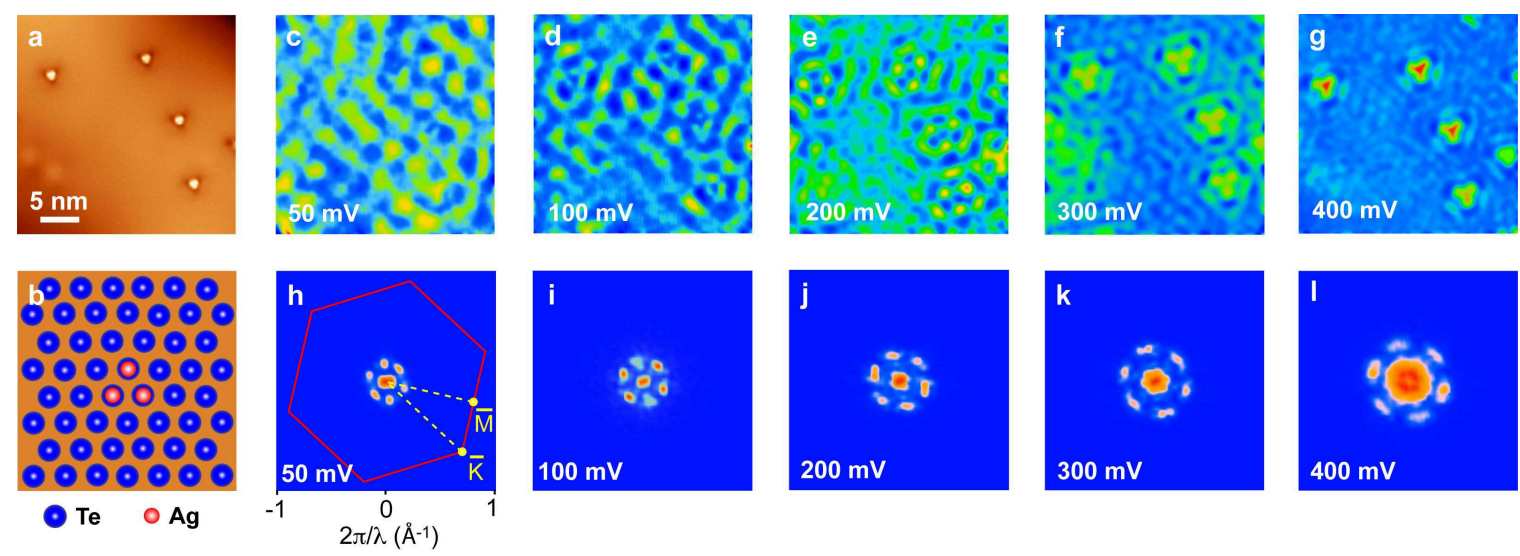

FIG. 2: Standing waves induced by $\mathrm{Ag}$ trimmers on $\mathrm{Bi}_{2} \mathrm{Te}_{3}(111)$ surface. (a) STM image (28 nm by $28 \mathrm{~nm}$ ) of a region with four $\mathrm{Ag}$ trimmers adsorbed on $\mathrm{Bi}_{2} \mathrm{Te}_{3}(111)$ surface. (b) The adsorption geometry of $\mathrm{Ag}$ trimmer. (c) to (g) The $d I / d V$ maps of the same area as (a) at various sample bias voltages. Imaging conditions: $\mathrm{I}=0.1 \mathrm{nA}$. Each map has 128 by 128 pixels and took two hours to complete. The interference fringes are evident in the images. The green and red regions indicate modulations with high intensity. (h) to (k) The FFT power spectra of the $d I / d V$ maps in (c) to (g). The SBZ in (h) is superimposed on the power spectra to indicate the directions in $\vec{q}$-space. The resolution of FFT, which is $2 \pi / 28 \mathrm{~nm}^{-1}$, is determined by the size of the STM image.

intensity in the $\bar{\Gamma}-\overline{\mathrm{K}}$ directions vanishes (see supporting material 23]). Such phenomena can be understood by exploring possible scattering processes on the CEC in the reciprocal space [Fig. 3(a)]. Generally, the $\vec{k}_{i}$ and $\vec{k}_{f}$ pairs with high joint DOS should dominate the quantum interference. For energies at which the interference fringes are prominent, the regions on CEC with high DOS are primarily centered around the $\bar{\Gamma}-\overline{\mathrm{K}}$ directions [10]. Therefore, three scattering wave vectors, labeled $\vec{q}_{1}, \vec{q}_{2}$ and $\vec{q}_{3}$, might be expected to appear in the power spectra. Among them, however, only $\vec{q}_{2}$ is along the $\bar{\Gamma}-\bar{M}$ directions and can generate the observed standing waves. Both $\vec{q}_{1}$ and $\vec{q}_{3}$ are invisible in the power spectra. There is a simple argument to account for the disappearance of $\vec{q}_{1}$ : the time-reversal invariance. The topological states $|\vec{k} \uparrow\rangle$ and $|-\vec{k} \downarrow\rangle$ are related by the timereversal transformation: $|-\vec{k} \downarrow\rangle=\mathcal{T}|\vec{k} \uparrow\rangle$, where $\mathcal{T}$ is the time-reversal operator. It is straightforward to show that $\langle-\vec{k} \downarrow|U| \vec{k} \uparrow\rangle=-\langle\vec{k} \uparrow|U|-\vec{k} \downarrow\rangle^{*}=-\langle-\vec{k} \downarrow|U| \vec{k} \uparrow\rangle=0$ for fermions, where $U$ is a time-reversal invariant operator and represents the impurity potential of the nonmagnetic Ag impurity. Therefore, the backscattering between $\vec{k}$ and $-\vec{k}$ is quantum-mechanically prohibited. Most of the observed features in the interference pattern, including the extinction of wave vector $\vec{q}_{3}$, have been recently well explained by a full theoretical treatment [27] based on the T-matrix approach for multiband systems [28]. In addition to the existence of standing waves, the absence of backscattering represents the second and most striking aspect of our experiment, which makes the topological standing waves more extraordinary as compared to the conventional surface states on metal samples [17, 18, 19, 20].

We can obtain the dispersion of the massless Dirac

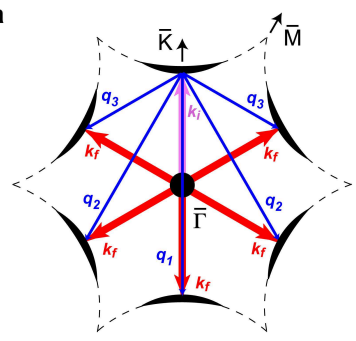

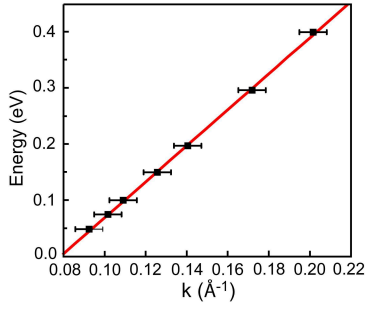

FIG. 3: (a) The scattering geometry. The CEC is in the shape of a hexagram. The dominant scattering wave vectors connect two points in $\bar{\Gamma}-\overline{\mathrm{K}}$ directions on CEC. $\vec{k}_{i}$ (pink arrow) and $\vec{k}_{f}$ (red arrows) denote the wave vectors of incident and scattered states. $\overrightarrow{q_{1}}, \overrightarrow{q_{2}}$ and $\overrightarrow{q_{3}}$ (blue arrows) are three possible scattering wave vectors. (b) Energy dispersion as a function of $k$ in the $\bar{\Gamma}-\overline{\mathrm{K}}$ direction. The data (black squares) are derived from FFT in Fig. 2. The red line shows a linear fit to the data with $v_{F}=4.8 \times 10^{5} \mathrm{~m} / \mathrm{s}$. The error bars represent the resolution of FFT (see the caption of Fig. 2).

fermions in the $\bar{\Gamma}-\overline{\mathrm{K}}$ direction using the interference patterns and their Fourier transforms. For $\vec{q}_{2}$, the scattering geometry determines $q_{2}=\sqrt{3} k$ [see Fig. 3(a)], where $k$ is the wave vector in the $\bar{\Gamma}-\overline{\mathrm{K}}$ direction at a given energy. The resulting $k$ values vary linearly with energy [Fig. 3(b)]. The slope of the linear fitting provides a measurement of the Dirac fermion velocity $v_{F}$, which is $4.8 \times 10^{5} \mathrm{~m} / \mathrm{s}$. In addition, the energy of the Dirac point is estimated to be $0.25 \mathrm{eV}$ by the intercept of the dispersion with the energy axis. These observations are in agreement with the results from the first-principles calculation and the ARPES data [7, 22]. More importantly, the unoccupied states, which are inaccessible to ARPES, 
can be probed by the standing waves with STM.

Interference fringes are also found at the step edges on the surface [29] [Figs. 4(a) to 4(h)]. Similar to the case of $\mathrm{Ag}$ trimmers, the standing waves produced by steps are predominantly propagating along the $\bar{\Gamma}-\bar{M}$ direction. The fringes are clearly visible even at the negative bias voltages probably owing to the stronger scattering potential compared to that of the Ag trimmers. The dispersion curve deduced from these patterns again shows a linear relation between the scattering wave vector and the energy [Fig. 4(i)]. Using the slope of the linear fitting together with the same scattering geometry as that for the Ag trimmer, the Fermi velocity is found to be $4.8 \times 10^{5}$ $\mathrm{m} / \mathrm{s}$, the same as that obtained from the standing waves caused by the Ag impurities.
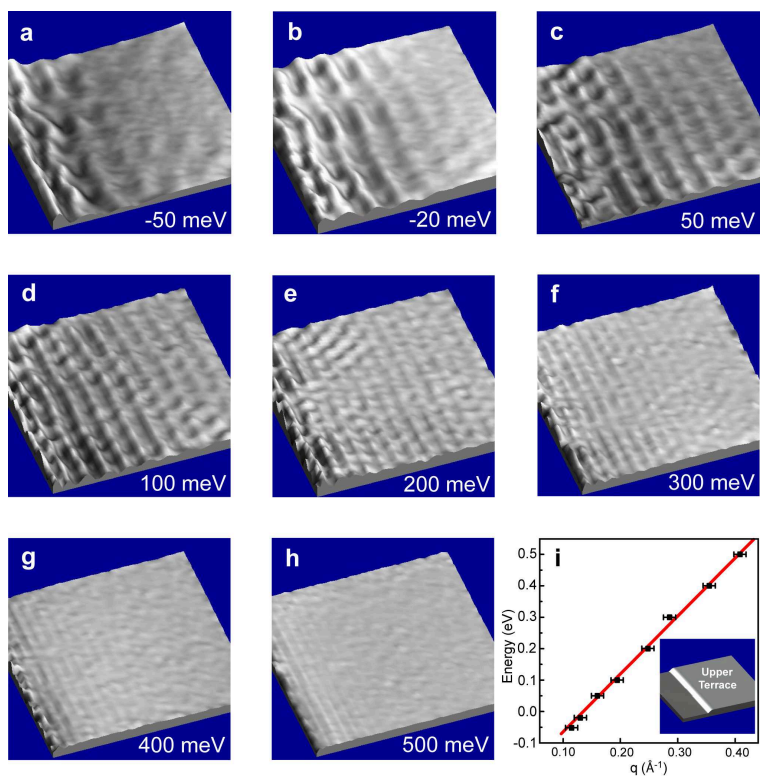

FIG. 4: Standing waves on the upper terrace by a step edge ((a) to (h)). All the images are $d I / d V$ maps at various bias voltages of an area of $35 \mathrm{~nm}$ by $35 \mathrm{~nm}$. Imaging conditions: $\mathrm{I}=0.1 \mathrm{nA}$. (i) Energy dispersion deduced from the standing waves at the step edge. The dispersion is a function of the scattering wave vector $q$. The inserted STM image shows the step that produces the standing waves in (a) to (h).

The existence of standing wave strongly supports the surface nature of topological states. An important issue that immediately arises is whether the topological states respond differently to the magnetic and the nonmagnetic impurities. Theoretically, it was pointed out [5, 30, 31] that a time-reversal breaking perturbation, such as magnetic impurities, can induce scattering between the states $|\vec{k} \uparrow\rangle$ and $-|\vec{k} \downarrow\rangle$ and open up a local energy gap at the Dirac point. It remains an open question to observe the distinct signature of time-reversal breaking in topological insulators.

We thank S.-C. Zhang, X.-L. Qi, Y. Ran and S.-Q. Shen for valuable discussions. The work is supported by NSFC and the National Basic Research Program of China. The STM topographic images were processed using WSxM (www.nanotec.es).

Note added. At the completion of this manuscript for submission, we became aware of related work by P. Rousan et al. [32]. The authors reported STM study of scattering from disorder in BiSb alloy.

* Electronic address: xc@mail.tsinghua.edu.cn

† Electronic address: qkxue@mail.tsinghua.edu.cn

[1] B. A. Bernevig, T. L. Hughes, and S.-C. Zhang, Science 314, 1757 (2006).

[2] M. König et al., Science 318, 766 (2007).

[3] L. Fu, C. L. Kane, and E. J. Mele, Phys. Rev. Lett. 98, 106803 (2007).

[4] J. E. Moore and L. Balents, Phys. Rev. B 75, 121306 (2007).

[5] X.-L. Qi, T. L. Hughes, and S.-C. Zhang, Phys. Rev. B 78, 195424 (2008).

[6] J. C. Y. Teo, L. Fu, and C. L. Kane, Phys. Rev. B 78, 045426 (2008).

[7] H. J. Zhang et al., Nature Phys. 5, 438 (2009).

[8] D. Hsieh et al., Nature 452, 970 (2008).

[9] D. Hsieh et al., Science 323, 919 (2009).

[10] Y. L. Chen et al., Science 325, 178 (2009).

[11] D. Hsieh et al., Nature 460, 1101 (2009).

[12] Y. Xia et al., Nature Phys. 5, 398 (2009).

[13] Y. S. Hor et al., Phys. Rev. B 79, 195208 (2009).

[14] X.-L. Qi, R. Li, J. Zang, and S.-C. Zhang, Science 323, 1184 (2009).

[15] L. Fu and C. L. Kane, Phys. Rev. Lett. 100, 096407 (2008).

[16] X.-L. Qi, T. L. Hughes, S. Raghu, and S.-C. Zhang, Phys. Rev. Lett. 102, 187001 (2009).

[17] M. F. Crommie, C. P. Lutz, and D. M. Eigler, Nature 363, 524 (1993)

[18] M. F. Crommie, C. P. Lutz, and D. M. Eigler, Science 262, 218 (1993).

[19] Y. Hasegawa and Ph. Avouris, Phys. Rev. Lett. 71, 1071 (1993).

[20] G. A. Fiete and E. J. Heller, Rev. Mod. Phys. 75, 933 (2003).

[21] J. E. Hoffman et al., Science 297, 1148 (2002).

[22] Y. Y. Li et al. (unpublished).

[23] See EPAPS for additional materials about the experimental results.

[24] S. Urazhdin et al., Phys. Rev. B 66, 161306 (2002).

[25] S. Urazhdin et al., Phys. Rev. B 69, 085313 (2004).

[26] The other candidate model of the Ag trimmer is that the $\mathrm{Ag}$ atom substitutes a topmost layer Te atom. In this case, the formation of $\mathrm{Ag}$ trimmers is kinetically more difficult. Although the exact structure of $\mathrm{Ag}$ trimmers does not affect the main conclusion here, it remains an interesting subject for further study, for example, by firstprinciples calculation.

[27] W.-C. Lee, C. J. Wu, D. P. Arovas, and S.-C. Zhang, arXiv:0910.1668 (2009).

[28] W.-C. Lee and C. J. Wu, arXiv:0906.1973 (2009).

[29] Z. Alpichshev et al., arXiv:0908.0371 (2009). 
[30] Q. Liu, C.-X. Liu, C. Xu, X.-L. Qi, and S.-C. Zhang, Phys. Rev. Lett. 102, 156603 (2009).

[31] X.-L. Qi, T. L. Hughes, and S.-C. Zhang, Nature Phys.
4, 273 (2008).

[32] P. Rousan et al., Nature 460, 1106 (2009). 